\title{
Outcomes of infectious syphilis in pregnant patients and maternal factors associated with congenital syphilis diagnosis, Alberta, 2017-2020
}

\author{
Jennifer Gratrix ${ }^{1}$, Jennifer Karwacki ${ }^{1}$, Lynn Eagle ${ }^{1}$, Lindsay Rathjen ${ }^{1}$, Ameeta Singh², Angel Chu ${ }^{1,3}$, \\ Petra Smyczek ${ }^{1,2 \star}$
}

\begin{abstract}
Background: Congenital syphilis (CS) is a significant public health challenge, requiring early diagnosis and treatment to improve infant outcomes. The aim of this study is to describe public health outcomes of infectious syphilis cases among pregnant patients and factors associated with a CS diagnosis for their infant.

Methods: We conducted a retrospective review of demographic and clinical characteristics of infectious syphilis cases diagnosed during pregnancy and resulting infant outcomes in Alberta from 2017 to 2020 from the provincial communicable disease database. Adequate maternal treatment was defined as receiving at least one dose of Benzathine penicillin G-LA 2.4 million units IM at least 28 days before delivery. Univariate and multivariate analysis was performed to determine factors associated with CS diagnosis using SPSS version 25.
\end{abstract}

Results: A total of 374 cases of infectious syphilis were diagnosed in pregnancy, with two patients being diagnosed twice in a single pregnancy. The majority $(79.1 \% ; \mathrm{n}=296)$ of women had a live birth, followed by therapeutic abortion $(9.4 \% ; n=35)$, stillbirth $(7.5 \% ; n=28)$ and spontaneous abortion (4.0\%; $n=15)$. Infant records $(n=265)$ were available for review $(n=117$ CS cases and 148 non-cases). Correlates associated with CS were screening time in third trimester (adjusted odds ratio [AOR] 8.4, 95\% confidence interval [CI], 2.9-24.6) and fewer than 28 days before delivery (AOR 8.1, 1.4-47.8 [vs. first and second trimester] and inadequate treatment (AOR 86.1, Cl, 15.9-466.5). Among the CS cases, 23.1\% $(n=27)$ were stillborn compared with one $(0.7 \%)$ stillbirth in the non-CS infants $(p<0.001)$.

Conclusion: The early identification and treatment of syphilis in pregnancy is crucial to preventing poor infant outcomes.
This work is licensed under a Creative Commons Attribution 4.0 International License.

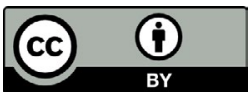

Affiliations

1 Sexually Transmitted Infections (STI) Services, Alberta Health Services, Edmonton, AB

2 Department of Medicine, University of Alberta, Edmonton, $A B$

${ }^{3}$ Department of Medicine, Cumming School of Medicine, Calgary, AB

\section{Correspondence:}

petra.smyczek@

albertahealthservices.ca

Suggested citation: Gratrix J, Karwacki J, Eagle L; Rathjen L, Singh AE, Chu A, Smyczek P. Outcomes of infectious syphilis in pregnant patients and maternal factors associated with congenital syphilis diagnosis, Alberta, 2017-2020. Can Commun Dis Rep 2022;48(2/3):61-7. https://doi.org/10.14745/ccdr.v48i23a02

Keywords: sexually transmitted infections, syphilis, congenital syphilis, prenatal screening

\section{Introduction}

Congenital syphilis (CS) is a worldwide public health challenge and reflects the incidence of infectious syphilis in the heterosexual female population (1). Untreated syphilis during pregnancy can profoundly affect pregnancy outcomes, resulting in spontaneous abortion, stillbirth, perinatal death and serious sequelae in infected infants.

The World Health Organization has set global targets for 2030 to reduce the incidence of syphilis by $90 \%$ and to reduce CS to 50 or fewer cases per 100,000 live births in $80 \%$ of countries (2). The estimated global CS rate in 2016 was 473 (range $=385-561$ ) per 100,000 live births and 661,000 (range $=538,000-784,000$ ) total CS cases (3).

A provincial syphilis outbreak was declared in Alberta in 2016, after rates of infection doubled from 3.9/100,000 in 2014 to $8.8 / 100,000$ in 2015 and have climbed to 56.7/100,000 in 2020. During this time, the rate among women has increased 90 -fold 
from $0.6 / 100,000$ in 2014 to $49.6 / 100,000$ in 2020 (4). The proportion of pregnant cases climbed from $0 \%$ in 2014 to $15 \%$ in 2020 (unpublished data). From 2012 to 2016, only a single CS case had been reported compared with 121 CS cases reported from 2017 to 2020, with 45 and 56 cases diagnosed in 2019 and 2020, respectively (4). Twenty-eight infants were stillborn during this time.

The aim of this study is to describe the epidemiology of infectious syphilis cases diagnosed during pregnancy and to report pregnancy and neonatal outcomes.

\section{Methods}

A retrospective review was conducted of demographic and clinical characteristics of infectious syphilis cases diagnosed during pregnancy and resulting pregnancy and neonatal outcomes in Alberta (current population 4.3 million) from 2017 to 2020. In Alberta, prenatal syphilis screening is universally recommended during the first trimester, at delivery, and throughout pregnancy for those with ongoing risk $(5,6)$. All cases of syphilis are reportable by laboratories and clinicians to the provincial sexually transmitted infections (STI) program that is responsible for ensuring adequate treatment and partner notification of all confirmed cases. Partner notification nurses (PNN) act as case managers for patients by collaborating with the patient, the testing and treating healthcare providers, and the STI medical consultant to facilitate appropriate assessment, treatment and follow-up of patients and their sexual partners. All patients are assessed for pregnancy through patient or healthcare provider interviews by the PNN. For pregnant patients, the PNN also coordinates care with the delivery site to ensure serology at delivery and appropriate follow-up of infants.

Sexually transmitted infections medical consultants completed maternal staging of syphilis according to provincial case definitions (7) and reviewed infants for possible diagnoses with CS.

De-identified data were extracted from the provincial communicable disease database on June 25, 2021; mothers and infants were linked by unique record numbers. Variables extracted for each syphilis case included demographic, behavioural and clinical factors. Ethnicity, history of transactional sex, injection drug use (IDU), sex with a person who injects drugs (PWID) were self-reported during PNN interviews as part of routine public health follow-up and reporting requirements. A symptom inquiry for sores, rashes, lesions and neurological signs was completed through client self-report or by the healthcare provider. Correctional involvement included any case where the patient was initially diagnosed or treated in a correctional facility. Alberta is split into five geographic zones and these zones were assigned based on the patient's postal code. Healthcare proximity was defined based on population density and travel times to health services: metro/urban (municipalities with a population density of at least 20,000 per $\mathrm{km} 2$ and their adjacent communities with a tertiary or regional hospital); rural (population density between 100-10,000 per $\mathrm{km} 2$ ); and rural remote (population density fewer than 100 per $\mathrm{km} 2$ and more than $200 \mathrm{~km}$ to a regional centre). Public health contact was defined as patients contacted by PNN versus patients who PNN were unable to contact. Screening time was the difference between the delivery date and the collection date of the first positive specimen and then stratified into three categories: first and second trimester (at least 91 days); third trimester (28-90 days), and fewer than 28 days prior to delivery as this timing was insufficient for adequate treatment. Adequate maternal treatment was defined as receiving at least one dose of Benzathine penicillin G-LA 2.4 million units IM or aqueous crystalline penicillin G 18-24 million units per day for at least 10 days and more than 28 days before delivery. Time to treatment was calculated from the date of the first positive test result to the initial dose of medication. For birth outcomes, stillbirth was defined as a fetal death after 20 weeks gestation and spontaneous abortion was fetal demise at or before 20 weeks gestation.

Descriptive analyses were completed for IDU, sex with a PWID and transactional sex; however, due to large proportions of missing data (25\%-31\%), these variables were excluded from additional analysis. To identify factors associated with CS, analyses were performed by comparing CS and non-CS cases. Univariate analyses were performed using Chi-square or Fisher's exact test for categorical variables and Mann-Whitney tests for continuous variables, excluding missing data. To identify independent factors associated with CS, multivariable logistic regression was performed. All variables significant at $p \leq 0.10$ at the univariate level were assessed for multicollinearity. The number of days to treatment and adequate treatment were found to be highly correlated, so number of days to treatment was not included in model building. Models were built using all significant variables and forward step-wise approach was found to have the best Hosmer-Lemeshow Goodness-of-Fit score. Analyses were completed using IBM SPSS Statistics 19 (IBM, Armonk, New York, United States).

\section{Results}

A total of 374 cases of infectious syphilis were diagnosed in pregnancy between January 1, 2017 and December 31, 2020, with two patients being reinfected during the same pregnancy. The majority of cases self-reported First Nations ethnicity, resided in the Edmonton zone, were from a metropolitan/urban area, were diagnosed with early latent syphilis, were contacted by a PNN, and had a live birth. All cases were HIV-negative. A significant proportion of women reported IDU $(21.0 \%$; $n=58 / 276)$, sex with a PWID (18.1\%; $n=47 / 259)$, and transactional sex (11.0\%; $n=31 / 281)$; however, these variables had high proportions of missing data (Table 1). 
Table 1: Maternal characteristics of infectious syphilis cases among pregnant patients in Alberta, 2017-2020 $(\mathrm{N}=374)$

\begin{tabular}{|l|r|r|}
\hline \multicolumn{1}{|c|}{ Characteristics } & N=374 & \multicolumn{2}{c|}{$\%^{a}$} \\
\hline $\begin{array}{l}\text { Median age at diagnosis (years, } \\
\text { IQR) }\end{array}$ & 26 & $22-30$ \\
\hline Ethnicity & 58 & 15.5 \\
\hline White & 228 & 61.0 \\
\hline First Nations & 36 & 9.6 \\
\hline Métis & 6 & 1.6 \\
\hline Other & 46 & 12.3 \\
\hline Unknown/missing & & \\
\hline Correctional facility & 342 & 91.4 \\
\hline No & 32 & 8.6 \\
\hline Yes & & \\
\hline
\end{tabular}

Geographic zone

\begin{tabular}{|l|r|r|}
\hline South & 2 & 0.5 \\
\hline Calgary & 16 & 4.3 \\
\hline Central & 30 & 8.0 \\
\hline Edmonton & 217 & 58.0 \\
\hline North & 109 & 29.1 \\
\hline
\end{tabular}

Healthcare proximity

\begin{tabular}{|l|r|r|}
\hline Metro/urban & 229 & 61.2 \\
\hline Rural & 103 & 27.5 \\
\hline Rural remote & 42 & 11.2 \\
\hline
\end{tabular}

\begin{tabular}{|l|r|r|}
\hline Diagnosis year & & \\
\hline 2017 & 15 & 4.0 \\
\hline 2018 & 66 & 17.6 \\
\hline 2019 & 145 & 38.8 \\
\hline 2020 & 148 & 39.6 \\
\hline
\end{tabular}

\section{Public health contact}

Client contacted

Unable to contact

$325 \quad 86.9$

\section{Syphilis stage}

\begin{tabular}{|l|r|r|}
\hline Primary & 114 & 30.5 \\
\hline Secondary & 21 & 5.6 \\
\hline Early latent & 238 & 63.6 \\
\hline Early neurosyphilis & 1 & 0.3 \\
\hline Median days to treatment ${ }^{\text {b }}$ (IQR) & 6 & $3-13$ \\
\hline Birth outcome & \multicolumn{2}{|c|}{} \\
\hline Live birth & 296 & 79.1 \\
\hline Stillbirth & 28 & 7.5 \\
\hline Spontaneous abortion & 15 & 4.0 \\
\hline Therapeutic abortion & 35 & 9.4 \\
\hline
\end{tabular}

Abbreviation: IQR, interquartile range

a This table represents 374 cases of infectious syphilis among 372 pregnancies: two patients were

reinfected during the same pregnancy

${ }^{b}$ Five cases had no treatment record at time of diagnosis
Of the 324 cases with a live birth or stillbirth, infant records were available for all but three cases. Seven sets of twins were born, resulting in a total of 328 infant records for review. Nearly one-third $(35.7 \%$; $n=117)$ of the infants were diagnosed with CS, $45.1 \%$ ( $n=148)$ of infants did not meet CS case definitions (non-CS) and 19.2\% ( $n=63)$ of infants remain unstaged and are continuing follow-up. Maternal characteristics associated with CS in univariate analysis were no contact with PNN, having two or more partners in the last year, screening time in third trimester or fewer than 28 days before delivery, and not receiving adequate treatment (Table 2 and Table 3). Of the total 265 cases, one-third $(35.8 \% ; n=95)$ of cases were screened less than a month before delivery, with many of these $(25.3 \% ; n=67)$ being within two days of delivery. The majority $(61.1 \% ; n=162)$ of patients received adequate treatment for the prevention of CS; 161 patients received Benzathine penicillin G-LA 2.4 million units IM at least 28 days prior to delivery and one patient received aqueous crystalline penicillin $\mathrm{G} 24$ million units per day for 14 days. The remaining $39.2 \%$ ( $n=104)$ of patients were not treated adequately. One-quarter $(24.5 \% ; n=65)$ of patients were treated with at least a single dose of Benzathine penicillin G-LA 2.4 million units IM at or post-delivery, $11.3 \%(n=30)$ were treated $1-27$ days prior to delivery, two $(0.8 \%)$ cases received doxycycline $100 \mathrm{mg}$ b.i.d. for 14 days post-delivery, and one case received insufficient treatment with aqueous crystalline penicillin G 5 million units intravenously in a single dose post-delivery. An additional five cases (1.9\%) had no treatment record at the time of analysis. Correlates that remained independently associated with CS upon multivariable analysis were screening time in third trimester (adjusted odds ratio [AOR] 8.4, 95\% confidence interval $[\mathrm{Cl}], 2.9-24.6)$ and fewer than 28 days before delivery (AOR 8.1, 1.4-47.8 [vs. first and second trimester], and inadequate treatment (AOR 86.1, $\mathrm{Cl}, 15.9-466.5)$. Among the CS cases, $23.1 \%(n=27 / 117)$ were stillborn compared with one $(0.7 \%)$ stillbirth in the non-CS infants $(p<0.001)$. Despite diagnosis during the first and second trimester of nine CS cases, four cases were treated within fewer than 28 days before delivery. Of the 237 live births, all CS cases received treatment. Thirty (20.4\%) of the non-CS cases also received treatment; these cases did not meet Alberta case definition for CS (8) but were treated at the time of delivery based on clinical judgement and maternal history known at the time of admission after assessment by paediatric Infectious Diseases specialists. 
Table 2: Univariate analysis of maternal characteristics associated with infant outcomes from infectious syphilis cases among pregnant patients in Alberta, 2017-2020 ( $N=265)$

\begin{tabular}{|c|c|c|c|c|c|c|c|c|c|}
\hline \multirow{2}{*}{ Maternal characteristics } & \multicolumn{2}{|c|}{ Congenital } & \multicolumn{2}{|c|}{ Non-cases } & \multicolumn{2}{|c|}{ Total } & \multirow{2}{*}{$p$-value } & \multicolumn{2}{|c|}{ Unadjusted OR } \\
\hline & $(n=117)$ & $\%$ & $(n=148)$ & $\%$ & $(\mathrm{~N}=265)$ & $\%$ & & Rate & $95 \% \mathrm{Cl}$ \\
\hline Median age at diagnosis (years, IQR) & 27 & $23-31$ & 26 & $21-30$ & 26 & $22-31$ & 0.18 & 1.0 & $1.0-1.1$ \\
\hline \multicolumn{10}{|l|}{ Ethnicity } \\
\hline White & 17 & 14.5 & 24 & 16.2 & 41 & 15.5 & 0.37 & Ref & Ref \\
\hline First Nations & 71 & 60.7 & 96 & 64.9 & 167 & 63.0 & - & 1.0 & $0.5-2.1$ \\
\hline Métis & 13 & 11.1 & 7 & 4.7 & 20 & 7.5 & - & 2.6 & $0.9-8.0$ \\
\hline Other & 1 & 0.9 & 3 & 2.0 & 4 & 1.5 & - & 0.5 & $0.1-4.9$ \\
\hline Unknown/missing & 15 & 12.8 & 18 & 12.2 & 33 & 12.5 & - & 1.2 & $0.5-3.0$ \\
\hline \multicolumn{10}{|l|}{ Correctional facility } \\
\hline No & 107 & 91.5 & 133 & 89.9 & 240 & 90.6 & 0.66 & Ref & Ref \\
\hline Yes & 10 & 8.5 & 15 & 10.1 & 25 & 9.4 & - & 0.8 & $0.4-1.9$ \\
\hline \multicolumn{10}{|l|}{ Geographic zone } \\
\hline Calgary & 4 & 3.4 & 5 & 3.4 & 9 & 3.4 & 0.05 & Ref & Ref \\
\hline Central & 16 & 13.7 & 6 & 4.1 & 22 & 8.3 & - & 3.3 & $0.7-16.7$ \\
\hline Edmonton & 63 & 53.8 & 90 & 60.8 & 153 & 57.7 & - & 0.9 & $0.2-3.4$ \\
\hline North & 34 & 29.1 & 47 & 31.8 & 81 & 30.6 & - & 0.9 & $0.2-3.6$ \\
\hline \multicolumn{10}{|l|}{ Healthcare proximity } \\
\hline Metro/urban & 65 & 55.6 & 93 & 62.8 & 158 & 59.6 & 0.09 & Ref & Ref \\
\hline Rural & 39 & 33.3 & 32 & 21.6 & 71 & 26.8 & - & 1.7 & $1.0-3.1$ \\
\hline Rural remote & 13 & 11.1 & 23 & 15.5 & 36 & 13.6 & - & 0.8 & $0.4-1.7$ \\
\hline \multicolumn{10}{|l|}{ Diagnosis year } \\
\hline 2017 & 5 & 4.3 & 9 & 6.1 & 14 & 5.3 & 0.01 & 1.6 & $1.2-2.1$ \\
\hline 2018 & 16 & 13.7 & 43 & 29.1 & 59 & 22.3 & - & - & - \\
\hline 2019 & 44 & 37.6 & 52 & 35.1 & 96 & 36.2 & - & - & - \\
\hline 2020 & 52 & 44.4 & 44 & 29.7 & 96 & 36.2 & - & - & - \\
\hline \multicolumn{10}{|l|}{ Public health contact } \\
\hline Client contacted & 89 & 76.1 & 136 & 91.9 & 225 & 84.9 & $<0.001$ & Ref & Ref \\
\hline Unable to contact & 28 & 23.9 & 12 & 8.1 & 40 & 15.1 & - & 3.6 & $1.7-7.4$ \\
\hline \multicolumn{10}{|l|}{ Symptoms $^{\mathrm{a}}$} \\
\hline No & 49 & 48.0 & 64 & 44.8 & 113 & 46.1 & 0.61 & Ref & Ref \\
\hline Yes & 53 & 52.0 & 79 & 55.2 & 132 & 53.9 & - & 0.9 & $0.5-1.5$ \\
\hline \multicolumn{10}{|l|}{ Number of partners in last 12 months } \\
\hline Fewer than 2 & 21 & 17.9 & 10 & 6.8 & 31 & 11.7 & 0.005 & Ref & Ref \\
\hline At least 2 & 96 & 82.1 & 138 & 93.2 & 234 & 88.3 & - & 0.3 & $0.2-0.7$ \\
\hline \multicolumn{10}{|l|}{ Maternal syphilis stage } \\
\hline Primary & 34 & 29.1 & 43 & 29.1 & 77 & 29.1 & 1.00 & 1.0 & $0.6-1.7$ \\
\hline Secondary & 8 & 6.8 & 9 & 6.1 & 17 & 6.4 & - & 1.1 & $0.4-3.2$ \\
\hline Early latent & 75 & 64.1 & 95 & 64.2 & 170 & 64.2 & - & Ref & Ref \\
\hline Early neurosyphilis & 0 & 0 & 1 & 0.7 & 1 & 0.4 & - & - & $\begin{array}{r}0 \text { cell } \\
\text { precluded } \\
\text { regression } \\
\text { analysis }\end{array}$ \\
\hline Median days to treatment ${ }^{b}$ & 5 & $2.0-8.5$ & 6 & $\begin{array}{l}3.0- \\
10.0\end{array}$ & 6 & $3.0-10.0$ & 0.03 & 1.0 & $0.999-1.01$ \\
\hline
\end{tabular}


Table 2: Univariate analysis of maternal characteristics associated with infant outcomes from infectious syphilis cases among pregnant patients in Alberta, 2017-2020 ( $N=265)$ (continued)

\begin{tabular}{|c|c|c|c|c|c|c|c|c|c|}
\hline \multirow{2}{*}{ Maternal characteristics } & \multicolumn{2}{|c|}{ Congenital } & \multicolumn{2}{|c|}{ Non-cases } & \multicolumn{2}{|c|}{ Total } & \multirow{2}{*}{$p$-value } & \multicolumn{2}{|c|}{ Unadjusted OR } \\
\hline & $(n=117)$ & $\%$ & $(n=148)$ & $\%$ & $(\mathrm{~N}=265)$ & $\%$ & & Rate & $95 \% \mathrm{Cl}$ \\
\hline \multicolumn{10}{|l|}{ Screening time } \\
\hline First to second trimester & 9 & 7.7 & 111 & 75.0 & 120 & 45.3 & $<0.001$ & Ref & Ref \\
\hline Third trimester & 18 & 15.4 & 32 & 21.6 & 50 & 18.9 & - & 6.9 & $2.8-16.9$ \\
\hline At least 28 days from delivery & 90 & 76.9 & 5 & 3.4 & 95 & 35.8 & - & 222 & $71.9-685.9$ \\
\hline \multicolumn{10}{|l|}{ Adequate treatment } \\
\hline No & 99 & 84.6 & 4 & 2.7 & 103 & 38.9 & $<0.001$ & 198.0 & $65.0-602.7$ \\
\hline Yes & 18 & 15.4 & 144 & 97.3 & 162 & 61.1 & - & Ref & Ret \\
\hline
\end{tabular}

Abbreviations: $\mathrm{Cl}$, confidence interval; IQR, interquartile range; OR, odds ratio; Ref, reference; -, no applicable

axcludes 20 cases with missing data

Excludes six cases with missing treatment date

Table 3: Multivariate analysis of maternal characteristics associated with infant outcomes from infectious syphilis cases among pregnant patients in Alberta, 2017-2020 $(\mathrm{N}=\mathbf{2 6 5})$

\begin{tabular}{|c|c|c|c|}
\hline $\begin{array}{c}\text { Maternal } \\
\text { characteristics }\end{array}$ & $\begin{array}{l}\text { Adjusted } \\
\text { OR }\end{array}$ & $95 \% \mathrm{Cl}$ & $p$-value \\
\hline \multicolumn{4}{|l|}{ Screening time } \\
\hline $\begin{array}{l}\text { First to second } \\
\text { trimester }\end{array}$ & Ref & - & - \\
\hline Third trimester & 8.4 & $2.9-24.6$ & $<0.001$ \\
\hline $\begin{array}{l}\text { At least } 28 \text { days from } \\
\text { delivery }\end{array}$ & 8.1 & $1.4-47.8$ & 0.02 \\
\hline \multicolumn{4}{|l|}{ Adequate treatment } \\
\hline Yes & Ref & - & - \\
\hline No & 86.1 & $15.9-466.5$ & $<0.001$ \\
\hline
\end{tabular}

\section{Discussion}

In Canada, the rates of infectious syphilis have been increasing sharply in the past five years; particularly alarming is an increase of infectious cases by $740 \%$ among females between 2016 and 2020 (8). Similar epidemiological trends have been seen in the United States with nearly 2,100 cases of CS in 2020 (9). Since the current syphilis outbreak started in 2015, Alberta has seen an unprecedented number of infectious syphilis cases among pregnant women resulting in CS cases and stillbirths. Inadequate maternal treatment was the most important predictor for a diagnosis of CS in our study. Several factors contributed to inadequate treatment during pregnancy. Firstly, the odds of giving birth to an infant with CS were eight times higher among patients screened in the third trimester and for patients screened in the last month of pregnancy as the late diagnosis leaves inadequate time for treatment. Furthermore, four of the nine CS cases with maternal screening during the first and second trimesters did not receive treatment four weeks prior to delivery due to barriers to care. Our findings are similar to those in a recent study on maternal syphilis treatment that showed that no pregnant patient with treatment in the first trimester delivered a neonatal CS case. Patients who initiated treatment in the third trimester had an increased risk of stillbirth, preterm birth and low birth weight (10). Another study examining the determinants associated with CS and adverse pregnancy outcomes found that every week of delay in treatment was related to 2.82-fold increased risk for adverse pregnancy outcomes (11).

While screening in the first trimester and at mid-gestation aims at preventing $\mathrm{CS}$, the goal of screening at delivery is early diagnosis and treatment of infants born to mothers with infectious syphilis. A cost effectiveness analysis in the United States in 2018 found that repeat screening in the third trimester is superior to single screening during the first trimester and is both cost-effective and results in improvement in maternal and neonatal outcomes (12). During the last infectious syphilis outbreak in Alberta in the mid-2000s, universal mid-gestation screening was introduced but, after a review of the Alberta prenatal screening program, it was discontinued in 2012 due to low uptake and limited utility for the diagnosis of additional new syphilis infections (13), although syphilis rates were lower at that time. Canadian and United States Centers for Disease Control and Prevention (CDC) syphilis screening guidelines recommend screening in the first trimester or at the first prenatal visit and repeat screening at 28-32 weeks' gestation and again at delivery for patients in areas with high rates of syphilis and for women at ongoing risk for syphilis acquisition $(14,15)$. Our study results support current Alberta syphilis screening guidelines for pregnant patients recommending universal maternal screening in the first trimester, at delivery $(5,6)$ and rescreening for those at ongoing risk. The majority of the mothers interviewed reported multiple partners in the last year and more than one-half reported symptoms, thereby meeting current recommendations for rescreening during pregnancy. Despite meeting current provincial screening recommendations for being at risk and requiring frequent re-screening throughout pregnancy, we found a high number of patients with an initial screen late in pregnancy (one-quarter within two days of delivery) and two cases of reinfection during the same pregnancy. We believe that frequent 
testing and re-testing of pregnant women up to monthly after an initial negative test result but who are at ongoing risk is an important tool to prevent further newborn syphilis cases. Increasing overall knowledge on syphilis and awareness of existing screening guidelines among medical providers are important steps to improve health outcomes of pregnant women and their infants. Opportunistic screening when women at risk present to healthcare for non-pregnancy related causes can lead to earlier diagnosis and treatment thereby reducing morbidity and mortality related to CS. Use of point of care testing and symptomatic syphilis treatment should be considered for women at risk for being lost to follow up. Offering screening to women in non-traditional care settings, such as addiction treatment centers, correctional facilities or emergency room departments, can further increase screening among women otherwise not engaging in prenatal care. The high proportion of cases in our study diagnosed in the third trimester underlines the need for non-traditional approaches for testing and treatment, as many patients who access services late in pregnancy are affected by adverse social determinants of health including poverty and mental health and addictions issues. In addition, a significant proportion of patients were Indigenous, demonstrating the need for culturally appropriate services. In response to the mid-2000s resurgence of infectious syphilis in Alberta, an outreach team in Edmonton geographical zone expanded to include registered nurses with the team of Indigenous community health representatives; offering culturally appropriate care. Outreach services can be a valuable strategy in reaching persons at risk for STIs since services are delivered to populations that would not normally be aware of or able to access services due to their life circumstances (16). Previous evaluations of outreach services in Edmonton, including the use of incentives, have highlighted the utility of these services in identifying new cases (17).

Nurse case management has been found to increase linkage to care and improve patient outcomes $(18,19)$. In our study population, patients without public health contact had worse outcomes with a 3.6-fold increased risk of giving birth to an infant with CS. Overall, the median time from first specimen collection to initiating treatment was six days and only five cases remained untreated. We hypothesize that our treatment success is related to the case management role that PNNs play in client engagement and prioritization of pregnant patients and their sexual contacts.

\section{Limitations}

This is the first Canadian study describing outcomes of infants born to patients with infectious syphilis during pregnancy. One possible limitation of our study is that our reported cases may underestimate the number of pregnant patients with syphilis, especially with the reduction of health services and patients choosing not to access care during the coronavirus disease 2019 pandemic, as well as cases that PNNs were unable to contact. Additionally, since we used retrospective data collected for surveillance purposes, data on behavioural characteristics, like
IDU and transactional sex, was missing in a significant proportion of our study population, which may be related to underlying stigma and social desirability bias, and therefore underestimate their impact.

\section{Conclusion}

Our study shows that early identification of syphilis in pregnancy through adherence to prenatal screening guidelines and a strong public health program to link patients to timely care are key in the prevention of CS cases. As syphilis rates increase and infections spread to rural and remote areas with limited access to health and social programs, it is imperative that sufficient resources for public health follow-up are available to facilitate the engagement of patients in care. In addition, a review of current screening practices in combination with increased awareness not only among members of the healthcare team but also in the general public may be required to respond to the changing epidemiology of syphilis, particularly to the increased prevalence of syphilis in young heterosexual populations. Lastly, we need to continue to engage with and work with affected communities to deliver services in a culturally and societally appropriate way.

\section{Authors' statement}

JG - Contributed to the concept of this report, performed statistical analysis, contributed to acquisition of data, contributed to interpretation of data, revising the manuscript critically for important intellectual content, and approved the final version PS - Contributed to the concept of this report, drafted the initial manuscript, contributed to interpretation of data, revising the manuscript critically for important intellectual content, and approved the final version

JK - Contributed to acquisition of data

$L E$, LB, AES, AC - Contributed to interpretation of data, revising the manuscript critically for important intellectual content, and approved the final version

The content and view expressed in this article are those of the authors and do not necessarily reflect those of the Government of Canada.

\section{Competing interests}

None.

\section{Acknowledgements}

We would like to acknowledge the tireless efforts of the members of the provincial STI Services program in Alberta.

\section{Funding}

None. 


\section{References}

1. Rac MW, Revell PA, Eppes CS. Syphilis during pregnancy: a preventable threat to maternal-fetal health. Am J Obstet Gynecol 2017;216(4):352-63. DOI PubMed

2. World Health Organization. Global Health Sector Strategy on Sexually Transmitted Infections 2016-2021. Geneva (CH): WHO; 2016. https://www.who.int/reproductivehealth/ publications/rtis/ghss-stis/en/

3. Korenromp EL, Rowley J, Alonso M, Mello MB, Wijesooriya NS, Mahiané SG, Ishikawa N, Le LV, Newman-Owiredu M, Nagelkerke N, Newman L, Kamb M, Broutet N, Taylor MM. Global burden of maternal and congenital syphilis and associated adverse birth outcomes-Estimates for 2016 and progress since 2012. PLoS One 2019;14(2):e0211720. DOI PubMed

4. Government of Alberta. Interactive Health Data Application. Edmonton (AB): Government of Alberta; 2021.

http://www.ahw.gov.ab.ca/IHDA_Retrieval/redirectToURL. do?cat $=81 \&$ subCat $=466$

5. Government of Alberta. Alberta Prenatal Screening Guidelines for Select Communicable Diseases. Edmonton (AB): Government of Alberta; 2018. https://open.alberta.ca/dataset/0ac7acb6-dc90-41338f63-5946d4bbf4d1/resource/782751ed-17b9-4116-9aa4227e55ec0299/download/alberta-prenatal-screeningguidelines-2018-10.pdf

6. Government of Alberta. Treatment Guidelines for Sexually Transmitted Infections (STI) in Adolescents and Adults, 2018. Edmonton (AB): Government of Alberta; 2018. https://open.alberta.ca/dataset/93a97f17-5210-487da9ae-a074c66ad678/resource/bc78159b-9cc4-454e$8 d c d-c c 85 e 0 f c c 435 / d o w n l o a d / s t i-t r e a t m e n t-g u i d e l i n e s-$ alberta-2018.pdf

7. Government of Alberta. Alberta Health Public Health Notifiable Disease Management Guidelines Syphilis. Edmonton (AB): Government of Alberta; 2021. https://open.alberta.ca/publications/syphilis

8. Public Health Agency of Canada. Infectious Syphilis and Congenital Syphilis in Canada, 2020 (infographic). Ottawa (ON): PHAC; (updated 2021; accessed 2022-01-22). https://www.canada.ca/en/public-health/services/ publications/diseases-conditions/infectious-syphiliscongenital-syphilis-canada-2020.html

9. Centers for Disease Control and Prevention. Congenital Syphilis: Preliminary 2020 Data (accessed 2022-01-21). https://www.cdc.gov/std/statistics/2020/Congenital-SyphilispreliminaryData.htm

10. Wan Z, Zhang H, Xu H, Hu Y, Tan C, Tao Y. Maternal syphilis treatment and pregnancy outcomes: a retrospective study in Jiangxi Province, China. BMC Pregnancy Childbirth 2020;20(1):648. DOI PubMed
11. Oin JB, Feng TJ, Yang TB, Hong FC, Lan LN, Zhang CL, Liu XL, Yang YZ, Xiao SY, Tan HZ. Synthesized prevention and control of one decade for mother-to-child transmission of syphilis and determinants associated with congenital syphilis and adverse pregnancy outcomes in Shenzhen, South China. Eur J Clin Microbiol Infect Dis 2014;33(12):2183-98. DOI PubMed

12. Hersh AR, Megli CJ, Caughey AB. Repeat Screening for Syphilis in the Third Trimester of Pregnancy: A Cost-Effectiveness Analysis. Obstet Gynecol 2018;132(3):699-707. DOI PubMed

13. Plitt SS, Osman M, Sahni V, Lee BE, Charlton C Simmonds K. Examination of a prenatal syphilis screening program, Alberta, Canada: 2010-2011. Can J Public Health 2016;107(3):e285-90. DOI PubMed

14. Public Health Agency of Canada. Syphilis guide: Key information and resources. Ottawa (ON): PHAC; 2020. https://www.canada.ca/en/public-health/services/infectiousdiseases/sexual-health-sexually-transmitted-infections/ canadian-guidelines/syphilis.html

15. Workowski KA, Bachmann LH, Chan PA, Johnston CM, Muzny CA, Park I, Reno H, Zenilman JM, Bolan GA. Sexually Transmitted Infections Treatment Guidelines, 2021. MMWR Recomm Rep 2021;70(4):1-187. DOI PubMed

16. National Collaborating Centre for Infectious Diseases. Outreach with Vulnerable Populations. Winnipeg (MB): NCCID; 2011. https://nccid.ca/publications/outreach-withvulnerable-populations/

17. Gratrix J, Fan S, Anderson B, Conroy P, Hewitt S, Parnell T, Jaipual J, Parker P, Singh AE, Talbot J. The Use of Financial Compensation as an Incentive for Infectious Syphilis Case Finding among Vulnerable Populations in Edmonton, Canada. Proceedings of the 19th Biennial Conference of the International Society for Sexually Transmitted Diseases Research; 2011 July 10-13; Quebec City, Canada. Sexually Transm Infect 2011;87(Suppl 1): Poster P5-S4.05. https://sti.bmj.com/content/87/Suppl_1

18. Starbird LE, Han HR, Sulkowski MS, Budhathoki C, Reynolds NR, Farley JE. Care2Cure: A randomized controlled trial protocol for evaluating nurse case management to improve the hepatitis $C$ care continuum within HIV primary care. Res Nurs Health 2018;41(5):417-27. DOI PubMed

19. Starbird LE, Budhathoki C, Han HR, Sulkowski MS, Reynolds NR, Farley JE. Nurse case management to improve the hepatitis $\mathrm{C}$ care continuum in HIV co-infection: results of a randomized controlled trial. J Viral Hepat 2020;27(4):376-86. DOI PubMed 